\title{
Experimental optimal cloning of four-dimensional quantum states of photons
}

\author{
E. Nagali, ${ }^{1}$ D. Giovannini, ${ }^{1}$ L. Marrucci, ${ }^{2,3}$ S. Slussarenko, ${ }^{2}$ E. Santamato, ${ }^{2}$ and F. Sciarrino, 4, * \\ ${ }^{1}$ Dipartimento di Fisica dell'Università "La Sapienza", Roma 00185, Italy \\ ${ }^{2}$ Dipartimento di Scienze Fisiche, Università di Napoli "Federico II", \\ Compl. Univ. di Monte S. Angelo, 80126 Napoli, Italy \\ ${ }^{3}$ CNR-SPIN, Compl. Univ. di Monte S. Angelo, 80126 Napoli, Italy \\ ${ }^{4}$ CNR-Istituto Nazionale di Ottica, Firenze, Italy
}

\begin{abstract}
Optimal quantum cloning is the process of making one or more copies of an arbitrary unknown input quantum state with the highest possible fidelity. All reported demonstrations of quantum cloning have so far been limited to copying two-dimensional quantum states, or qubits. We report the experimental realization of the optimal quantum cloning of four-dimensional quantum states, or ququarts, encoded in the polarization and orbital angular momentum degrees of freedom of photons. Our procedure, based on the symmetrization method, is also shown to be generally applicable to quantum states of arbitrarily high dimension - or qudits - and to be scalable to an arbitrary number of copies, in all cases remaining optimal. Furthermore, we report the bosonic coalescence of two single-particle entangled states.
\end{abstract}

Classical information can be freely measured, perfectly copied on demand, and broadcast without fundamental limitations. The handling of quantum information, which is encoded in the quantum states of physical systems, is instead subject to several fundamental restrictions. For example, an unknown quantum state of an individual system cannot be measured completely, unless we have infinite identical copies at our disposal. For a finite number of copies $N$, the state estimation can only be partial, and it can be characterized by an average "fidelity" lower than one (where one corresponds to perfect state identification). It has been proven that the optimal value of such state estimation fidelity is given by $F_{\text {est }}^{d}(N)=(N+1) /(N+d)$, where $d$ is the dimension of the quantum space [1]. A similar restriction is posed by the quantum no-cloning theorem, stating that an unknown quantum state cannot be copied perfectly [2]. It is however possible to make imperfect copies, characterized by a cloning fidelity lower than one [3]. Starting with $N$ identical copies of the input state and generating $M>N$ output optimal copies, the optimal copying fidelity is given by $F_{\text {clon }}^{d}(N, M)=\frac{M-N+N(M+d)}{M(N+d)}$, for the case of "symmetric" cloning, that is for a uniform fidelity of all copies [4]. It is important to note that, for a given input, the optimal cloning fidelity is always higher than the corresponding optimal state-estimation fidelity, reducing to the latter in the limit $M \rightarrow \infty$ [5]. Therefore, the optimal quantum cloning process is useful whenever one needs to broadcast quantum information among several parties without measuring it in the process. Quantum cloning thus represents an important multipurpose tool of the emerging quantum information technology. Let us stress that the advantage of quantum cloning over state estimation grows for an increasing dimension $d$ of the quantum state. More specific applications of quantum cloning are found in the security assessment of quantum cryptography, the realization of minimal disturbance measurements, the enhancement of the transmission fi- delity over a lossy quantum channel, and the separation of classical and quantum information $[6,7]$.

It is well known that all tasks of quantum information can be performed using only two-dimensional quantum states, or qubits. However, it has been recently recognized that significant fundamental and practical advantages can be gained by employing higher dimensional quantum states instead, or qudits. For example, quantum cryptographic protocols based on qudits may achieve improved security, entangled qudits can show increased resistance to noise, a qudit-based quantum computation may require less resources for its implementation, and the use of quantum computing as physics simulators can be facilitated by using qudits [8-14].

Light quantum states can be used for implementing qudits, either by exploiting many-photon systems [1517] or by combining different degrees of freedom of the same photon ("hybrid" states) such as linear momentum, arrival time, and orbital angular momentum (OAM) or other transverse modes [18-21]. In particular, we have recently reported the first experimental generation and tomography of hybrid qudits with dimension $d=4$, also dubbed ququarts, that were encoded in the polarization and OAM of single photons [22].

In this paper, we report the realization of the optimal quantum cloning $1 \rightarrow 2$ (i.e., $N=1, M=2$ ) of ququarts $(d=4)$ encoded in the polarization and OAM of single photons. The cloning process is based on the symmetrization technique [23-25], that has been recently proven theoretically to be optimal for arbitrary dimension $d$ [26]. The simultaneous control of polarization and OAM was made possible thanks to the q-plate, a photonic device introducing a spin-orbit angular momentum coupling [27, 28].

Let us recall the working principle of the symmetrization method for $1 \rightarrow 2$ quantum cloning, in the case of a generic $d$-dimensional quantum state (qudit) [2326]. The input qudit $|\varphi\rangle_{s}$ is sent into one input port 


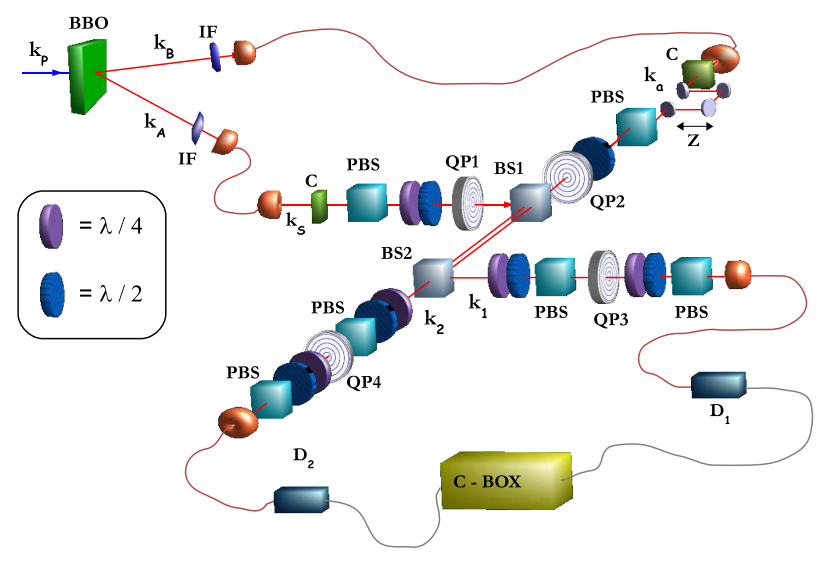

FIG. 1: Experimental apparatus for implementing the $1 \rightarrow$ 2 optimal quantum cloning of polarization-OAM photon ququarts. See main text for a definition of all symbols.

(mode $\mathbf{k}_{s}$ ) of a balanced beam splitter (BS). In the other BS input port (mode $\mathbf{k}_{a}$ ) we send an ancilla photon in the fully mixed random $d$-dimensional state $\rho_{a}=$ $\frac{I_{d}}{d}=\frac{1}{d} \sum_{n}|n\rangle_{a}\langle n|$. The basis is here chosen such that $|1\rangle \equiv|\varphi\rangle$. After the interaction in the BS, we consider only the case of the two photons emerging in the same output mode. The cloning fidelity is defined as the average overlap between the quantum state of each output photon emerging from the BS and the input photon state $|\varphi\rangle$. Hence, we can distinguish two cases, depending on the ancilla state: (i) the input state is $|1\rangle_{s}|1\rangle_{a}$ or (ii) it is any of the other $d-1$ states $|1\rangle_{s}|n\rangle_{a}$ with $n \neq 1$. In the first case, Hong-Ou-Mandel (HOM) quantum interference due to the bosonic symmetry leads to a doubled probability of having the two photons emerging in a common output BS mode, as compared to the second case. So, once given that the two photons emerge in the same mode (i.e., successful cloning occurs), then the first case has a relative probability of $2 /(d+1)$ while the second case has a total probability $(d-1) /(d+1)$. Since the first case corresponds to a fidelity of 1 (both photons are identical to the input one) and the second of 0.5 (one photon is identical, the other is orthogonal), we obtain the following average cloning fidelity $F=\frac{1}{2}+\frac{1}{d+1}$, that corresponds just to the upper bound for the cloning fidelity $F_{c l o n}^{d}(N, M)$ given above, for $N=1$ and $M=2$.

This cloning procedure has been previously demonstrated experimentally only for photonic qubits, either encoded in the polarization space $\pi$ [24] or in the bidimensional OAM subspace $o_{2}$ spanned by $m= \pm 2$ [26], where $m$ is the OAM eigenvalue per photon along the beam axis in units of $\hbar$. We now consider photonic ququarts encoded in the four-dimensional "spinorbit" space $\pi \otimes o_{2}$, i.e. obtained as tensor product of the polarization space and the OAM subspace with $m= \pm 2$. A generic separable state in this space will be indicated as $|\varphi, \ell\rangle=|\varphi\rangle_{\pi}|\ell\rangle_{O_{2}}$, where $|\cdot\rangle_{\pi}$ and $|\cdot\rangle_{O_{2}}$ stand for the polarization and OAM quantum states, respectively. We introduce in this space a first basis $\left\{\left|1_{\mathrm{I}}\right\rangle,\left|2_{\mathrm{I}}\right\rangle,\left|3_{\mathrm{I}}\right\rangle,\left|4_{\mathrm{I}}\right\rangle\right\}$, hereafter called "logic" basis, corresponding to $\{|R,+2\rangle,|R,-2\rangle,|L,+2\rangle,|L,-2\rangle\}$, where $R$ $(L)$ refers to right (left) circular polarization and the \pm 2 integers refer to the OAM eigenvalue. Given this first basis, we may introduce four other bases such that they are all mutually unbiased to each other [29]. In our spin-orbit space $\pi \otimes o_{2}$, all the states belonging to the five mutually unbiased basis can be generated and detected by an appropriate combination of birefringent wave plates, polarizing beam-splitters, q-plates and single mode fibers, as described in Ref. [22]. Three of these bases (bases I, II, and III) are formed of separable states of polarization and OAM of the photon (e.g., basis I is the logical one defined above), while the remaining two (bases IV and $\mathrm{V}$ ) are formed of entangled states of these two degrees of freedom (see [22] for the complete list of states). In particular, the IV basis, which will be utilized in the present work, is composed of the following four states: $(|R,+2\rangle \pm|L,-2\rangle) / \sqrt{2}$ and $(|L,+2\rangle \pm|R,-2\rangle) / \sqrt{2}$.

We now consider the optimal quantum cloning of a photonic ququart in the spin-orbit space $\pi \otimes o_{2}$. Being $d=4$, we expect an optimal cloning fidelity $F=7 / 10$. If $|\varphi\rangle=|1\rangle$ is the input state, each output cloned photon (tracing out the state of the other photon) is hence expected to be found in the mixed state:

$$
\rho_{1}=\rho_{2}=\frac{1}{10}(7|1\rangle\langle 1|+| 2\rangle\langle 2|+| 3\rangle\langle 3|+| 4\rangle\langle 4|),
$$

where the set $\{|1\rangle,|2\rangle,|3\rangle,|4\rangle\}$ forms a basis in the space $\pi \otimes O_{2}$ (not necessarily the logical one). In particular, we will experimentally test the outcome of the quantum cloning procedure for all the four states of the logical basis I and for all the four states of the IV basis, corresponding to entangled spin-orbit states.

The experimental layout is schematically reported in Fig. 1. A $\beta$-barium borate crystal (BBO) cut for type-II phase matching, pumped by the second harmonic of a Ti:Sapphire mode-locked laser beam, generates via spontaneous parametric fluorescence photon pairs on modes $k_{A}$ and $k_{B}$ with linear polarization, wavelength $\lambda=795$ $\mathrm{nm}$, and pulse bandwidth $\Delta \lambda=4.5 \mathrm{~nm}$, as determined by two interference filters (IF). The coincidence rate of the source is equal to $18 \mathrm{kHz}$. Photons generated on mode $k_{A}$ and $k_{B}$ are delivered to the setup via single mode fibers, thus defining their transverse spatial modes to a pure $\mathrm{TEM}_{00}$, corresponding to OAM $m=0$. After the fiber output, two wave plates $(\mathrm{C})$ compensate the polarization rotation introduced by the fibers and a polarizing beam-splitter (PBS) projects the polarization on the horizontal state $|H\rangle_{\pi}$. Then on mode $k_{s}$ the ququart to be cloned is encoded in the single photon polarization 
and OAM through a ququart preparation stage, based on a combination of wave plates, a q-plate, a PBS (only for bases I, II, III), and additional wave plates (see Ref. [22] for details). On mode $k_{a}$, for quantum cloning the ancilla photon is prepared in a fully mixed state $\rho_{a}=\frac{I_{\pi}}{2} \frac{I_{02}}{2}$, i.e. fully randomized both in polarization and in OAM. This is obtained by randomly rotating, during each experimental run, a half-wave plate inserted before the q-plate QP2 and by randomly inserting or removing another halfwave plate located after the same q-plate. The time delay between photons on mode $k_{s}$ and $k_{a}$ was set to zero by an adjustable delay line $(\mathrm{Z})$, in order to ensure the interference condition necessary for the optimal quantum cloning process within the balanced beam splitter BS1. A second beam splitter (BS2) is then used to separate the two photons emerging from the same output port of BS1, allowing post-selection of this outcome by coincidence detection. On both output modes $k_{1}$ and $k_{2}$ of BS2 we perform a full ququart state measurement, by combining a standard polarization analysis set and an OAM analysis set, the latter based on the quantum transferrer $o_{2} \rightarrow \pi$ [28]. Depending on the specific ququart basis being used, the detailed setting of this ququart measurement stage varies slightly, as discussed in [22]. Finally, the output photons are coupled into single mode fibers and detected by single-photon counters $\left(\mathrm{D}_{1}\right.$ and $\left.\mathrm{D}_{2}\right)$ connected to the coincidence box (C-BOX) recording the time-coincident photon detections.

(a)

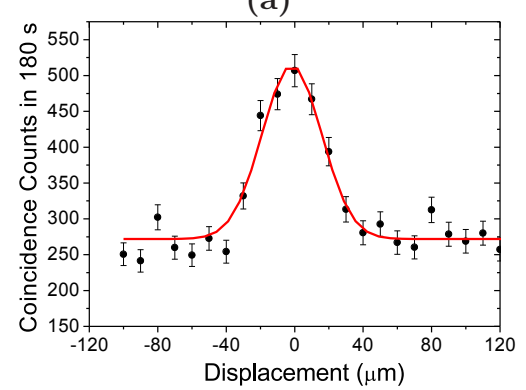

(b)

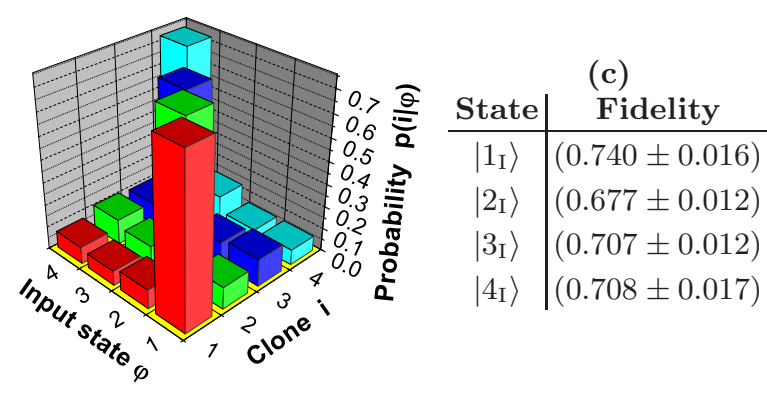

FIG. 2: Experimental results of the cloning process for the ququart states belonging to the I basis a) Hong-Ou-Mandel coalescence for two input photons prepared in state $|L,-2\rangle$. The enhancement peak is of $R=(1.90 \pm 0.13)$. b) Probability $p(i \mid \varphi)$ of detecting a clone in any output state $|i\rangle$ of the basis, for any given input state $|\varphi\rangle$ of the same basis. c) Experimental cloning fidelities for the four input states.
As a first step, we have verified the occurrence of the HOM interference between the two photon ququarts impinging on modes $k_{s}$ and $k_{a}$ of BS1. The ancillary photon was prepared in the same quantum state as the signal photon, in order for the interference to occur. The twophoton coincidence counts were measured as a function of the optical path delay between $k_{s}$ and $k_{a}$. In Fig. 2a we report an example of the results we obtained for the case of an input state belonging to the logical basis. The HOM peak is observed with a measured coincidence enhancement $R=(1.89 \pm 0.05)$, consistent with the theoretical value $R_{t h}=2$. Once ensured a good interference condition between the two photons, we moved on to testing the quantum cloning for each of the four input states of the logical basis. Being $|\varphi\rangle$ the input state to be cloned, the measurement stage on mode $k_{1}$ has been set so as to filter only outcoming photons in state $|\varphi\rangle$ while on mode $k_{2}$ all four possible outcomes $|i\rangle$ of the logical basis have been detected. We have thus recorded the corresponding coincidence counts $N_{\varphi, i}$. These coincidence counts give us an estimate of the probability $p(i \mid \varphi)$ of each photon clone to be found in any specific state $|i\rangle$ of the basis, with $i=1,2,3,4$, as a function of the input state $|\varphi\rangle$, regardless of the state of the other photon. In particular, we have $p(i \mid \varphi)=N_{\varphi, i} / N$ for $i \neq \varphi$ and $p(\varphi \mid \varphi)=\left(N_{\varphi, \varphi}+\sum_{i \neq \varphi} N_{\varphi, i}\right) / N$, where $N=N_{\varphi, \varphi}+2 \sum_{i \neq \varphi} N_{\varphi, i}$. The factor 2 appearing in the expression of $N$ takes into account the additional coincidences that would be detected by swapping the measurements performed on modes $k_{1}$ and $k_{2}$, for $i \neq \varphi$, that of course are equal to $N_{\varphi, i}$ in the average. The theoretical values for these probabilities are given by the corresponding coefficients in the clone density matrix in Eq. (1). The cloning fidelity is $F=p(\varphi \mid \varphi)$. The experimental results obtained when cloning all states of the logical basis are reported in Fig. 2-b,c. The measured values of the fidelity, as well as their average value $\bar{F}_{I}=(0.708 \pm 0.007)$, are all in good agreement with the theoretical prediction $F=0.7$.

Cloning only states belonging to the logical basis is clearly not enough to demonstrate the generality of our cloning procedure. We have therefore repeated the cloning experiment for all ququart states belonging to the IV basis, that includes spin-orbit entangled states. These states can be generated and analyzed by exploiting the q-plate capability of entangling and disentagling the polarization and the OAM degree of freedom of a photon. This required removing the first PBS on modes $k_{1}$ and $k_{2}$ and properly setting the orientation of all wave plates [22]. In Fig. 3 we report the experimental results of the HOM peak for one of the states and of the cloning of all states belonging to the IV basis. Fig.3-a, in particular, demonstrates interference between two single-particle entangled states. As expected, this measurement underlines how the bosonic coalescence of two particles is not tied to the indistinguishability of each individual degree 
(a)
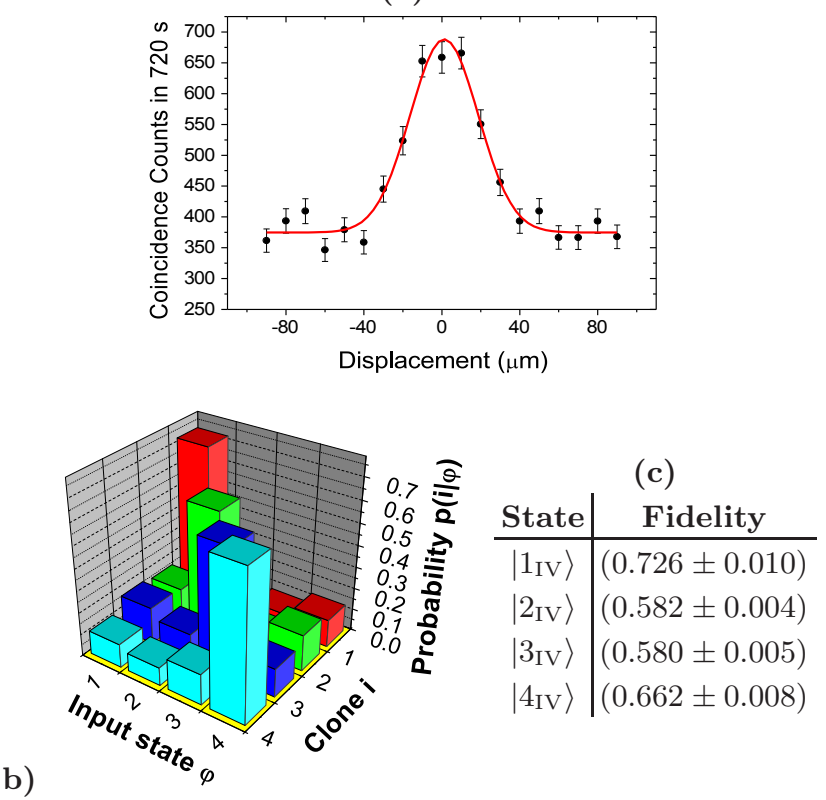

FIG. 3: Experimental results of the cloning process for all ququart states belonging to the IV basis, made of entangled spin-orbit states. a) Hong-Ou-Mandel coalescence for two input photons prepared in state $2^{-1 / 2}(|R,+2\rangle+|L,-2\rangle)$. The enhancement peak is of $R=(1.84 \pm 0.05)$. b) Probability $p(i \mid \varphi)$ of detecting a clone in an output state $|i\rangle$ of the IV basis, for any given input state $|\varphi\rangle$ of the same IV basis.c) Experimental cloning fidelities for all four input states.

of freedom, but rather that of the whole quantum state (whether each of such states is entangled, as is the case in our experiment, or separable). As can be inferred from the table in Fig. 3c, the cloning fidelities are again in reasonable agreement with the expected one, and the average fidelity value reads $\bar{F}_{I V}=(0.638 \pm 0.004)$. The small discrepancy with respect to the theoretical expectations is quantitatively well explained by the imperfect randomization of the ancilla photon (which is found to be somewhat unbalanced), the slightly lower HOM enhancement achieved $(R=1.84)$, and the non-unitary preparation and analysis fidelities $(\sim 0.9)$ [32]. We stress that the setup alignment has not been re-optimized for cloning states of basis IV, in order to properly test the universality of our cloning apparatus. We notice that the average value of the quantum cloning fidelity is much larger than the one expected for the quantum state estimation on a single copy, equal to 0.4 [5].

Finally, we note that the symmetrization procedure for the cloning of photonic qudits, that we have experimentally demonstrated here for the $1 \rightarrow 2$ case, can be scaled up to the general $N \rightarrow M$ cloning [33], that is starting with $N$ identical input photons and generating $M>N$ copies. The main idea, illustrated in Fig. 4, is that of using a cascaded configuration of $M-N$ beam splitters and $M-N$ ancilla photons in fully mixed states: Fig.4.

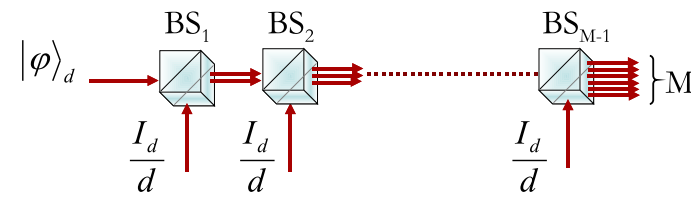

FIG. 4: Schematic representation of the $1 \rightarrow M$ quantum cloning process of a qudit state by cascading the symmetrization technique.

A more exhaustive demonstration of this result will be presented in a forthcoming paper.

In summary, we have implemented the optimal quantum cloning $1 \rightarrow 2$ of ququart states encoded in the polarization and OAM degrees of freedom of a single photon. This work was supported by project HYTEQ - FIRB, Finanziamento Ateneo 2009 of Sapienza Università di Roma, and european project PHORBITECH of the FET program (grant 255914).

* Electronic address: fabio.sciarrino@uniroma1.it

[1] N. Gisin, et al., Phys. Rev. Lett. 79, 2153 (1997).

[2] W.K. Wootters, et al., Nature 299, 802 (1982).

[3] V. Scarani, et al., Rev. Mod. Phys. 77, 1225 (2005).

[4] P. Navez, et al., Phys. Rev. A 68, 032313 (2003).

[5] D. Bruß, et al., Phys Lett A,253, 249 (1999).

[6] N. Gisin, et al., Rev. Mod. Phys. 74, 145 (2002).

[7] M. Ricci, et al., Phys. Rev. Lett. 95, 090504 (2005).

[8] D. Kaszlikowski, et al., Phys. Rev. Lett. 85, 4418 (2000).

[9] J. Cerf, et al., Phys. Rev. Lett. 88, 127902 (2002).

[10] D. Collins, et al., Phys. Rev. Lett. 88, 040404 (2002).

[11] M. Genovese, Phys. Rep. 413, 319 (2005).

[12] B. P. Lanyon, et al., Nature Phys. 5, 134 (2009).

[13] M. Neeley et al., Science 325, 722 (2009).

[14] T. Vértesi, et al., Phys. Rev. Lett. 104, 060401 (2010).

[15] E. V. Moreva, et al., Phys. Rev. Lett. 97, 023602 (2006).

[16] G. Vallone, et al., Phys. Rev. A 76, 012319 (2007).

[17] B. P. Lanyon, et al., Phys. Rev. Lett. 100, 060504 (2008).

[18] J. P. Torres, et al., Phys. Rev. A 67, 052313 (2003).

[19] A. Vaziri, et al., Phys. Rev. Lett. 91, 227902 (2003).

[20] G. Molina-Terriza, et al., Phys. Rev. Lett. 92, 167903 (2004).

[21] N. K. Langford, et al., Phys. Rev. Lett. 93, 053601 (2004).

[22] E. Nagali, et al., Phys. Rev. A 81, 052317 (2010).

[23] M. Ricci, et al., Phys. Rev. Lett. 92, 047901 (2004).

[24] F. Sciarrino, et al., Phys. Rev. A 70, 052305 (2004).

[25] W.T.M. Irvine, et al., Phys. Rev. Lett. 92, 047902 (2004).

[26] E. Nagali, et al., Nature Photon. 3, 720 (2009).

[27] L. Marrucci, et al., Phys. Rev. Lett. 96, 163905 (2006).

[28] E. Nagali, et al., Phys. Rev. Lett. 103, 013601 (2009).

[29] A. Klappenecker, et al., Lect. Notes Comput. Sci. 2984, 137 (2004).

[30] S. Franke-Arnold, et al., Laser \& Photon. Rev., 4, 299 (2008).

[31] G. Molina-Terriza, et al., Nature Phys. 3, 305 (2007).

[32] The weights of the four components of the ancilla mixed 
state in the basis IV have been experimentally found to be: $w_{1}=0.32, w_{2}=0.16, w_{3}=0.16$, and $w_{4}=0.36$. By considering the preparation and analysis fidelity and the factor $R$, we computed the expected fidelity values $F_{1}=0.702, F_{2}=0.594, F_{3}=0.597$, and $F_{4}=0.68$, with mean value $F_{I V}=0.645$, in good agreement with the experimental results.

[33] L. Masullo, et al., Phys. Rev. A 72, 060304 (2005). 